Check for updates

Cite this: RSC Adv., 2018, 8, 19238

\title{
High-throughput metabolomics used to identify potential therapeutic targets of Guizhi Fuling Wan against endometriosis of cold coagulation and blood stasis
}

\author{
Xiu-hong Wu, ${ }^{a}$ Chuang Zhao, ${ }^{a} \mathrm{Ai}-$ hua Zhang, ${ }^{\mathrm{a}}$ Jin-qi Zhang, ${ }^{a} \mathrm{Xu}$ Wang, ${ }^{a}$ \\ Xiao-lan Sun, ${ }^{a}$ Ze Sun ${ }^{a}$ and Xi-jun Wang (D) *abc
}

\begin{abstract}
Metabolomics is an emerging and robust discipline and involves the comprehensive evaluation of small molecule endogenous metabolites and enables the exploration of the pathogenesis of diseases. For example, endometriosis - a common disease which mostly occurs in women of childbearing age. A cure for endometriosis of cold coagulation and blood stasis (ECB) is highly sought after. This study was conducted to discover the potential biomarkers of ECB and the effective mechanism undertaken by Guizhi Fuling Wan (GFW) in treating ECB in rats. Urinary metabolomics were performed by using UPLCQ-TOF-MS with pattern recognition methods to evaluate the changes in metabolic profiles and to identify biomarkers for elucidating the mechanism of the treatment of ECB with GFW. The results showed that urinary metabolism in the two groups were distinctly separated on the $28^{\text {th }}$ day, and a total of 20 differential biomarkers ( 16 in the positive mode, 4 in the negative mode) were confirmed involving several key metabolic pathways which included phenylalanine, tyrosine and tryptophan biosynthesis, valine, leucine and isoleucine biosynthesis, glyoxylate and dicarboxylate metabolism, tyrosine metabolism and the citrate cycle. Following the oral administration of GFW, certain pathways were affected; these included the following: phenylalanine, tyrosine and tryptophan biosynthesis, valine, leucine and isoleucine biosynthesis, glyoxylate and dicarboxylate metabolism, tyrosine metabolism, citrate cycle, steroid hormone biosynthesis, tryptophan metabolism, phenylalanine metabolism, primary bile acid biosynthesis, and aminoacyl-tRNA biosynthesis. This study also demonstrated that the administration of GFW affected the levels of urine endogenous metabolites, thereby laying a foundation for further study of the pharmacodynamical mechanism of GFW.
\end{abstract}

Received 31st January 2018

Accepted 7th May 2018

DOI: $10.1039 / \mathrm{c} 8 \mathrm{ra00978c}$

rsc.li/rsc-advances

\section{Introduction}

Traditional Chinese medicine (TCM) plays a significant role in medical care in Asia, mainly for maintaining the general health in the body. ${ }^{1,2}$ It has been widely used in the treatment and prevention of disease. ${ }^{3}$ The use of TCM for clinical use follows the theory of TCM. The majority of herbal medicines exert synergistic interactions or reduce existing adverse reactions. ${ }^{4}$ The famous Chinese herbal formula Guizhi Fuling Wan (GFW),

${ }^{a}$ Sino-America Chinmedomics Technology Collaboration Center, National TCM Key Laboratory of Serum Pharmacochemistry, Chinmedomics Research Center of State Administration of TCM, Metabolomics Laboratory, Heilongjiang University of Chinese Medicine, Heping Road 24, Harbin 150040, China. E-mail: xijunwangls@ 126.com; Tel: $+86-451-82110818$

${ }^{b}$ State Key Laboratory of Quality Research in Chinese Medicine, Macau University of Science and Technology, Avenida Wai Long, Taipa, Macau, PR China

'National Engineering Laboratory for the Development of Southwestern Endangered Medicinal Materials, Guangxi Botanical Garden of Medicinal Plant, Nanning, Guangxi, China which was originally included in the "Correction of Errors in Medical Classics" composed by Qingren Wang in the Qing dynasty (A.D. 1830), was practiced in the clinic for approximately 200 years for the therapy of blood stasis syndrome in gynecological diseases including endometriosis, dysmenorrheal, amenorrhea, etc. GFW consists of five drugs: Cinnamomum cassia Blume, Poria cocos (Schw.) Wolf, Paeonia lactiflora Pall., Paeonia suffruticosa Andr., and Prunus persica (L.) Batsch. Although the Guizhi Fuling Wan have been proved to be effective in treating gynecological diseases, the treatment mechanism is not yet clear.

Endometriosis is a frequently-occurring and hormonedependent disease in women of child-bearing age. It typically causes dysmenorrheal, infertility, and chronic pelvic pain, affecting the reproductive health and immune system in females with endometriosis. Numerous immune cells are poorly regulated in females suffering from endometriosis including the neutrophils, dendritic cells, macrophages, natural killer cells, B cells and T helper cells. ${ }^{5-10}$ Furthermore, 
chemokines and cytokines, which are related to inflammation, angiogenesis and tissue growth, accumulate in the peritoneal fluid (PF) and plasma of females with endometriosis. ${ }^{11,12}$ Stimulating symptoms commonly profiled is skeptical of leading to the partial and systemic inflammatory milieu including pain and infertility. ${ }^{13-15}$ Cold coagulation blood stasis, a sub-type of blood stasis syndrome, is mainly caused by cold-evil. This syndrome can lead to other diseases including endometriosis and dysmenorrheal in women. The pathogenesis of cold coagulation blood stasis syndrome was recently found to be associated with changes in hemorheological properties including high blood viscosity, increased blood sedimentation, increased erythrocyte aggregation, reduced erythrocyte deformability, and coagulant function abnormality. ${ }^{16}$ Inflammatory factors and immune response were thought to be related closely to blood stasis syndrome. Inflammation and immune-related genes are prevalent in the gene expression profile of blood stasis syndromes, which may explain the reduction in function in immune response during the development and progression of the syndrome. ${ }^{17}$ Currently, the main treatments of endometriosis in Western countries are based on surgical and hormonerelated approaches. ${ }^{18}$ However, the relapse rate remains higher than $40 \%$ after 5 years. ${ }^{19,20}$ There has not been any significant findings that serve to improve this situation; thus, the purpose of this study is to explore the therapeutic mechanisms of GFW.

Metabolomics is a new branch of systemic biology which also includes genomics, transcriptomics, and proteomics. It can be utilized for researching the pathogenesis of TCM since it tracks the levels of endogenous metabolites. ${ }^{21}$ Metabolomics can be used to follow dynamic changes occurring in human or animal urine,,$^{22}$ blood, ${ }^{23}$ tissue extract ${ }^{24}$ and other biological metabolites. This in turn can be used to systematically analyze and elucidate the pathological processes occurring during infection or sickness as well as the pharmacology of traditional Chinese medicine and its mechanisms, i.e., to define the action sites, metabolic pathways and mechanism of action of the metabolites leading to internal body changes. ${ }^{25}$ Metabolites are closely related to phenotypic changes which provide the most effective method to elucidating the biomarkers in patients. ${ }^{26}$ In general, the two most commonly used analytical platforms in metabolomics research are nuclear magnetic resonance (NMR) spectroscopy and mass spectrometry (MS). ${ }^{27,28}$ Although NMR can provide unique compound structural information, it faces limitations in terms of sensitivity and resolution. By contrast, mass spectrometry remains the most common analytical technique in metabolomics research due to its high sensitivity and reproducibility. ${ }^{29,30}$ UPLC-MS is a widely used technique in metabolism research due to of its reproducibility and sensitivity. ${ }^{31,32}$ In metabolomics research, multivariate analytical methods such as principal components analysis (PCA), partial least squares-discriminant analysis (PLS-DA) and orthogonal single collection partial least squares-discriminant analysis (OPLS-DA), have been utilized. ${ }^{33}$ Urine metabolic profiling is the most common since urine collection is non-traumatic, and the profiles are able to provide various pieces of biochemical information. ${ }^{34}$ Highlights of this present study include the application of non-targeted urine metabolomics based on UPLC-Q/TOF-MS coupled with pattern recognition methods, for determining new potential biomarkers, making recommendations for interrelating metabolic pathways, and accounting for the mechanisms of action of GFW.

\section{Experimental methods}

\section{Materials and reagents}

The crude drugs: Cassia twig, Poria cocos, Peach kernel, Radix paeoniae rubra and Cortex moutan, were obtained from Harbin Tongrentang Drug Store (Harbin, China) and authenticated by Prof. Xijun Wang from the Heilongjiang University of Chinese Medicine. The estradiol benzoate injection was acquired from Animal Pharmaceutical Hangzhou, China (batch number 110202511). Sodium chloride injection was purchased from Harbin Medisan Pharmaceutical Co., LTD (batch number H23020611). Iodine volts disinfectant was purchased from LIRCON, Shandong (batch number 170321). HPLC grade acetonitrile was obtained from Fisher (USA). Deionized water was obtained from Watson's Food \& Beverage Co., Ltd. (Guangzhou, China). HPLC grade methanol was purchased from Fisher (USA). Formic acid was obtained from Tianjin Kermel Chemical Reagent Co., Ltd. (Tianjin, China). Leucine enkephalin was obtained from Sigma-Aldrich (MO, USA). The other reagents and chemicals used were of analytical grade.

\section{Preparation of GFW extract}

Cassia twig, Poria cocos, Peach kernel, Radix paeoniae rubra and Cortex moutan were mixed in a weight ratio of $1: 1: 1: 1: 1$. The mixture (100 g) was suspended in methanol (10 fold), and ultrasonic extraction was carried out over $30 \mathrm{~min}$. Following filtration, the residue was collected with 8 layers of gauze, before being decocted using methanol ( 8 fold) for $30 \mathrm{~min}$. The concentrated solution was then dried using a vacuum drying oven.

\section{Animal handing procedure and drug treatment}

Female Wistar rats (weighting $220 \pm 20 \mathrm{~g}$ ) were procured from Laboratory Animal Center of Heilongjiang University of Traditional Chinese Medicine. The room temperature and relative humidity were set at $25 \pm 1{ }^{\circ} \mathrm{C}$ and $40 \pm 5 \%$ respectively, with an alternating $12 \mathrm{~h}$ light/dark cycle. Water was given to the rats and normal food was fed ad libitum before the experiment. Before treatment, all animals in metabolism cages were left to acclimatize for a week. All rats were randomly put into 3 groups: the control group (sham group), the model group (surgery group), and the GFW group (treatment group), with 8 rats per group. The experimental procedures were approved by the Animal Care and Ethics Committee at Heilongjiang University of Chinese Medicine, and all experiments were performed in accordance with the Declaration of Helsinki.

In this paper, the rat model was established by autologous endometrial transplants and ice water immersion, using the endometriosis model which was previously described by Vernon and Wilson. ${ }^{35}$ All experimental procedures were executed 
during estrus. The modeling process was performed under sterile conditions. The rats in the model group and treatment group were anesthetized with $0.2 \mathrm{~mL} 100 \mathrm{~g}^{-1}$ sodium pentobarbital (2\%) and fastened in a supine position during estrous phase. Following the routine disinfection of the skin using $10 \%$ povidone-iodine solution, an approximately $2-3 \mathrm{~cm}$ midline incision was performed to enter the abdominal cavity. The left uterine horn was ligated on two sides, and a $1 \mathrm{~cm}$ sample was longitudinally cut open. The removed uterine tissue was placed in a sterile dish containing stroke-physiological saline solution before being cut into two pieces, each measuring $4 \times 4 \mathrm{~mm}$. Each section was sutured with USP 6/0 polyglactin (into the abdominal and uterine wall of the same rat). Prior to the closure of the abdominal wall, $2 \mathrm{~mL}$ of saline solution was administered in the peritoneal cavity. Subsequently, the midline incision was closed with a $3 / 0$ polyglactin suture. Following the surgery, the rats were intraperitoneally administered with gentamicin at a rate of $0.5 \mathrm{~mL} \mathrm{~d}^{-1}$ for 3 days. The rats were fed normally for 3 days following the surgery. Each rat was injected with estradiol $0.1 \mathrm{~mL}$ per day every 3 days three times in total. In the sham group, only the abdominal incision was performed; membrane transplantation was not carried out. Two weeks later, the rats in the model group and treatment group were put into ice water $\left(0-1{ }^{\circ} \mathrm{C}\right)$ for 8-10 min for 14 days. Following this, the GFW group was orally administrated with GFW (2.16 $\left.\mathrm{g} \mathrm{kg}^{-1}\right)$ whilst an aliquot of distilled water was intragastrically administrated to the sham group and model group. The oral administration procedure was continued for 28 days.

\section{Samples collection and preparation}

Each rat was placed into single metabolic cage and rat urine collected weekly. The urine was centrifuged at $13000 \mathrm{rpm}$ at $4{ }^{\circ} \mathrm{C}$ for $10 \mathrm{~min}$. The supernatants were then stored at $-80{ }^{\circ} \mathrm{C}$. Before analysis, the sample was thawed before being placed into a polypropylene tube after centrifugation at $13000 \mathrm{rpm}$ for $10 \mathrm{~min}$. The supernatant liquid was added to an aliquot volume of distilled water, vortex mixed for $30 \mathrm{~s}$, and centrifuged at $13000 \mathrm{rpm}$ for $10 \mathrm{~min}$, before the supernatants were filtered through a $0.22 \mu \mathrm{m}$ membrane filter. Finally, the supernatant was analyzed using LC/MS.

\section{H \& E staining}

For histopathological analysis, the ectopic endometrium of rats was fixed in $10 \%$ formaldehyde, embedded in paraffin and sectioned. The paraffin sections were fixed with $\mathrm{H} \& \mathrm{E}$ for routine examination. With the use of a microscope, the characteristics and diversity of tissue morphology among the groups was inspected.

\section{Metabolic profiling}

Chromatographic analysis was performed on an Acquity ${ }^{\mathrm{TM}}$ UPLC (Waters, Milford, USA) coupled with Masslynx (V4.1). Analysis was performed with an ACQUITY UPLC ${ }^{\mathrm{TM}}$ phenomenex column $(2.1 \mathrm{~nm} \times 50 \mathrm{~mm}, 1.7 \mu \mathrm{m})$ with auto-sampler at $4{ }^{\circ} \mathrm{C}$. The column temperature was kept at $40{ }^{\circ} \mathrm{C}$ and $0.4 \mathrm{~mL} \mathrm{~min}{ }^{-1}$ (flow rate). The optimal linear gradient system used a mobile phase consisting of a combination of (A) acetonitrile with $0.1 \%$ formic acid and (B), water with $0.1 \%$ formic acid. The gradient was set as follows: 0-8 min: 2-40\% A; 8-10 $\mathrm{min}$ : $40-98 \% \mathrm{~A} ; 10-$ 13 min: $98-100 \%$ A; $13-14$ min: $100-2 \%$ A; $14-17 \mathrm{~min}$, before maintaining $2 \%$ A for 3 min for equilibration of the column. 5 $\mu \mathrm{L}$ and $2 \mu \mathrm{L}$ urine samples were injected into the instrument for positive and negative mode, respectively. (electrospray ionization was conducted in both positive and negative modes). The analytical conditions were as follows: the ion source temperature was $110{ }^{\circ} \mathrm{C}$, and the desolvation gas flow and temperature were $750 \mathrm{~L} \mathrm{~h}^{-1}$ and $350{ }^{\circ} \mathrm{C}$, respectively. Capillary voltages of 1.3 $\mathrm{kV}$ (positive mode) and $1.5 \mathrm{kV}$ (negative mode) and sample cone voltages of $45 \mathrm{~V}$ (positive mode) and $50 \mathrm{~V}$ (negative mode) were preset. Mass spectrometry data was collected in centroid mode and the mass spectrum ranged from 100-1500 Da. To obtain accurate mass readings, a lock-mass of $0.2 \mathrm{ng} \mathrm{mL}{ }^{-1}$ leucine enkephalin was used to monitor ions in the positive mode $([\mathrm{M}+$ $\left.\mathrm{H}]^{+}=556.2771\right)$ and negative ion mode $\left([\mathrm{M}-\mathrm{H}]^{-}=554.2615\right)$ during integral analysis.

\section{Multivariate statistical analysis and data processing}

All data were imported into the Progenesis QI software (version 2.1, Waters Corporation, Milford, MA) for alignment and peakpicking. The ion intensity of each sample was normalized and imported into Ezinfo software 2.0 (Waters Corp.) and analyzed using the pattern recognition method which comprises principal components analysis (PCA), partial least-squared discriminant analysis (PLS-DA) and orthogonal partial leastsquares discriminant analysis (OPLS-DA). This enabled the generation of a data matrix which included the retention time, $\mathrm{m} / \mathrm{z}$, and the normalized peak area. The overview of the cluster of groups was analyzed in a principal using PCA. The OPLS-DA score enabled the acquisition of the variable importance of the projection (VIP) values and the relative intensities of the biomarkers following the analysis. Student's $t$-test was carried out to research the characteristics across groups.

\section{Biomarkers identification and metabolic pathway}

Exact MS data were initially utilized to verify the presence of certain metabolites based on their molecular masses. Based on the retention behavior (Rt) and exact masses $(\mathrm{m} / \mathrm{z})$ of the candidate biomarkers, elemental analysis was carried out using the precise mass and mass spectrometry data (including fragmentation patterns) to search the HMDB, KEGG, and Metlin databases. Massfragment ${ }^{\mathrm{TM}}$ embedded in the Masslynx analysis software was applied to characterize the MS/MS fragments. MetPA (Metabolomics Pathway Analysis) is the analysis software of metabolomics pathways and was applied to determine the most relevant metabolites pathways according to the analysis of the topological characteristics of the pathways. Using the KEGG graphics software package, the presence of the compounds was confirmed, and the pathway topology information was entered into a graph model. A relevant metabolic network map is created, with each node having a cross-over. Clicking on each node enables the retrieval of a detailed name and report. The related metabolic pathways of 
the potential biomarkers were then elucidated by MetaboAnalyst online software based on database sources including KEGG and HMDB.

\section{Statistical analysis}

Data is conveyed as the mean \pm SD for statistical analysis by SPSS version 21.0. By using a two-sample student's $t$-test, significant differences were tested and shown as average values with $p<0.05$ considered statistically significant, and $p<0.01$ represented a significant difference.

\section{Results}

\section{Morphological observation}

This is a preliminary study to observe the size of the ectopic endometrial tissue in rats using endometriosis and based on the spleen index. To calculate the implant volume, the dimensions (length, width, and height) were initially measured using a micrometer. Before the formula $(0.52 \times$ length $\times$ width $\times$ height) was applied. The endometriosis lesion tissue volume showed that a significant difference existed between the groups. The volumes of ectopic endometrial lesions with cystic vesicles in the model group was significantly greater. The cystic vesicles were transparent or translucent, and clear liquid was observed to have accumulated in the vesicles. However, the volumes of ectopic endometrial tissue in the GFW group were significantly smaller (or had disappeared) than those in the model group. The results are shown in Fig. 1.

\section{Histopathological observations}

The results of staining with $\mathrm{H} \& \mathrm{E}$ showed that the endometrial tissue was arranged more regularly and was lined with a long columnar epithelium. It also clearly showed the structure of the gland and that there were no obvious abnormalities of smooth muscle cells and uterine stromal. However, a gland cavity-like structure was observed in the graft, and the epithelial cells and interstitial cells in the inner wall of the grafts exhibited significant hyperplasia. Furthermore, the epithelial cells were incomplete, low columnar or flat, the cell structure was not clear, the interstitial more closely, and the blood supply was
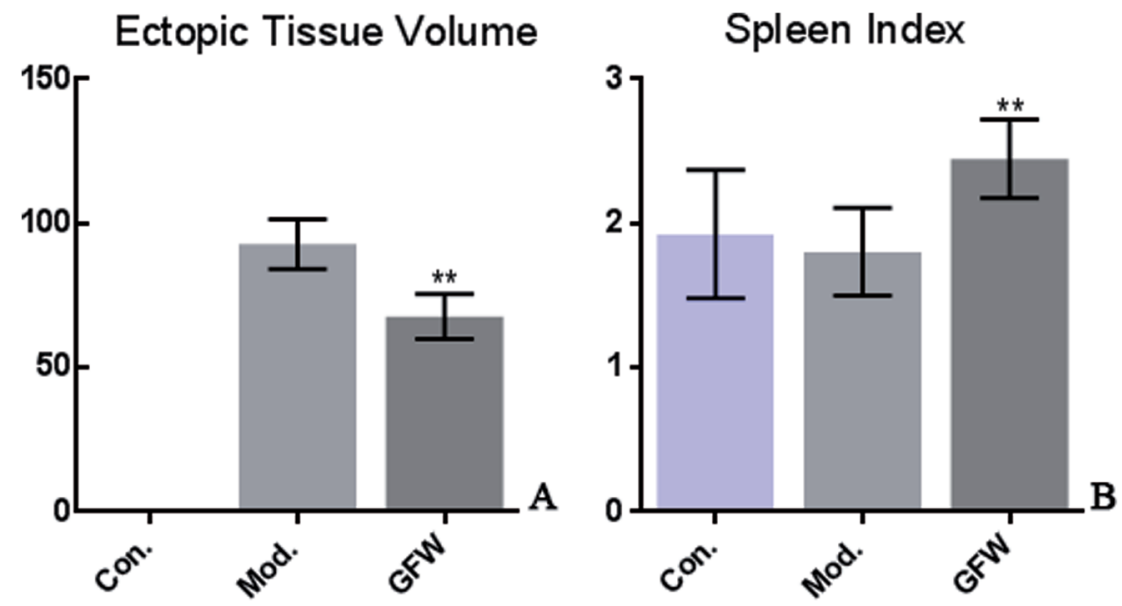

Fig. 1 The rat endometrial graft volume and spleen coefficient pictorial diagram in different groups after oral administration of GFW for 28 days. Data were expressed as means \pm SD. Significant differences when compared with the model group $* p<0.05, * * p<0.01$ (A): ectopic tissue volume; (B): the spleen index.

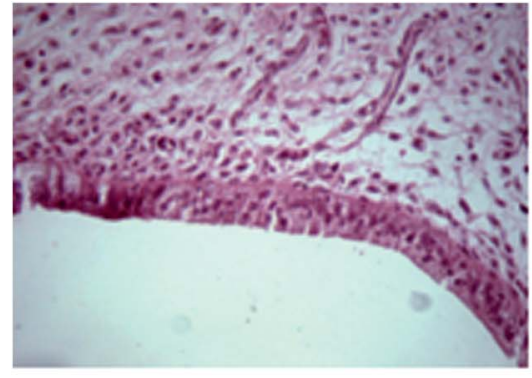

Con.

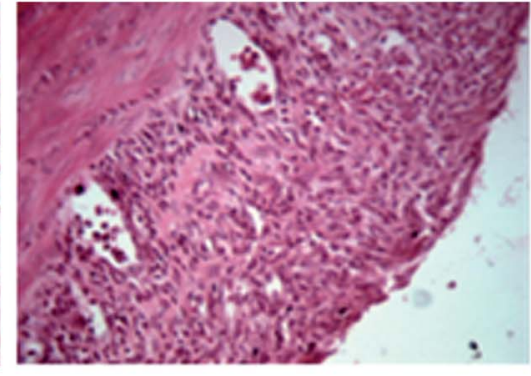

Mod.

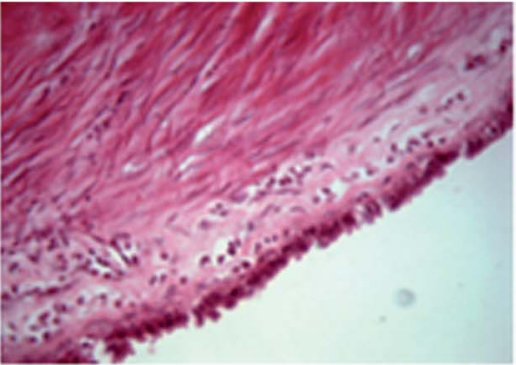

GFW

Fig. 2 Endometriosis effect of shape and structure of ectopic endometrial cells in the different groups after oral administration of GFW for 28 day (400 $\times$ magnification). 

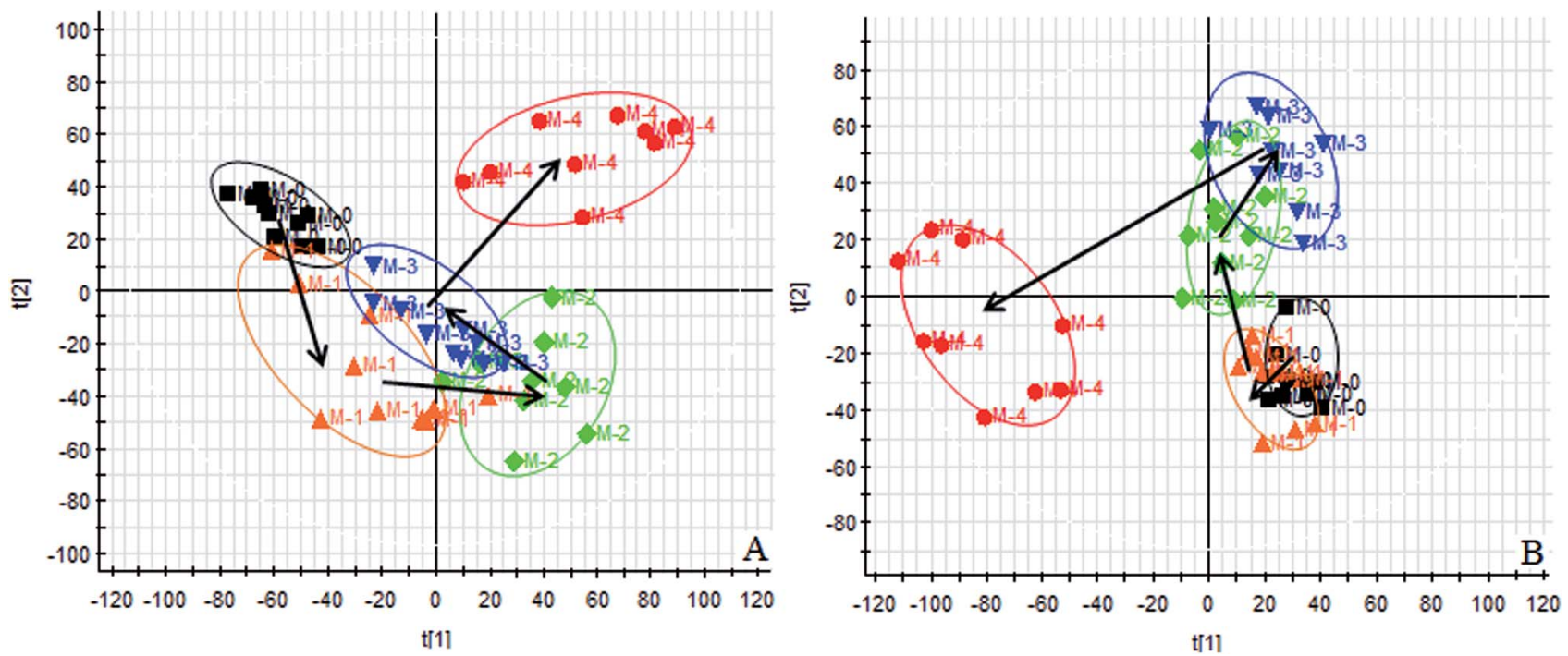

Fig. 3 Trajectory analysis of PCA score plot for urine profile in key time points of endometriosis model generation in positive and negative (A): the positive mode; (B): the negative mode; $\square$-0: con.; $\triangle$ M-1: mod-7 days; M-2: mod-14 days; $\nabla$ M-3: $\bmod -21$ days; $M-4$ : mod-28 days.
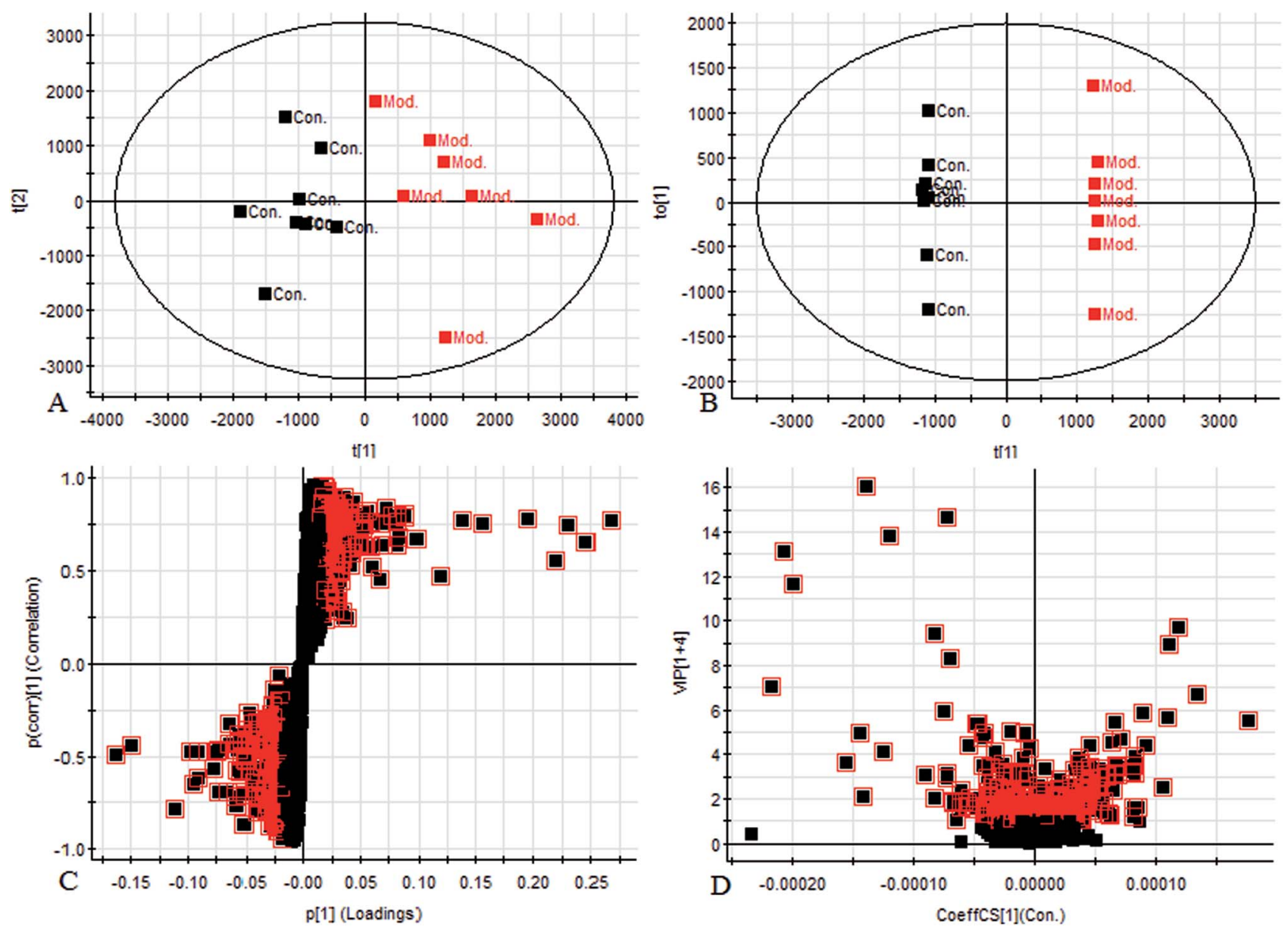

Fig. 4 Metabolomic profiling of endometriosis in positive mode at 28 days: (A) score plot of urine profile of control group and model group scanned by PCA analysis. (B) Score plot of urine profile of control group and model group scanned by OPLS-DA analysis. (C) VIP-plot of urine profile of control group and model group scanned by OPLS-DA analysis. (D) S-plot of urine profile of control group and model group scanned by OPLS-DA analysis. 

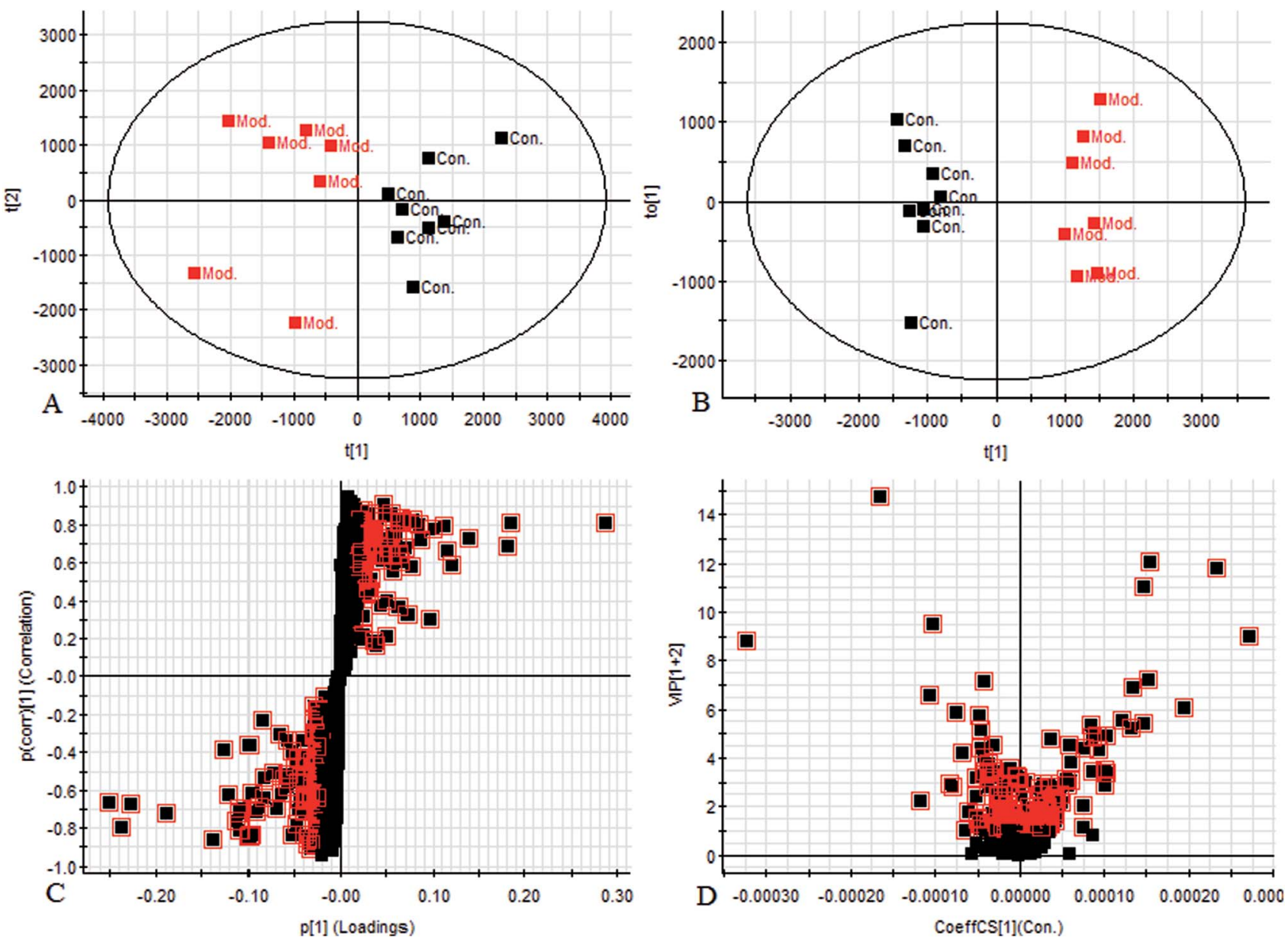

Fig. 5 Metabolomic profiling of endometriosis in negative mode at 28 days: (A) score plot of urine profile of control group and model group scanned by PCA analysis. (B) Score plot of urine profile of control group and model group scanned by OPLS-DA analysis. (C) VIP-plot of urine profile of control group and model group scanned by OPLS-DA analysis. (D) S-plot of urine profile of control group and model group scanned by OPLS-DA analysis.

abundant. By contrast with the model group, the abovementioned pathology of the administration group had improved as shown by a reduction in the epithelial cells in the cavity surface, a decrease in the number of interstitial cells, and a reduction in the number of blood vessels. It can be concluded that endometriosis was considerably improved following drug administration. The staining are shown in Fig. 2.

\section{Analysis of metabolite profiling}

Using the UPLC-MS conditions described above, urine samples were analyzed in both positive and negative modes. All the data were entered into Progenesis QI software for multiple statistical analyses. Generally, the trajectory analysis of PCA scores for the surgery model in both the positive (Fig. 3A) and negative mode (Fig. 3B) can report the differences between the models at key time points (0d, 7d, 14d, 21d, 28d). The PCA scores demonstrate that there appeared to be some grouping of the metabolic characteristics of 0 and 7 days but there were also intersections. However, these changed at the 28 day as a result of surgery, reaching maximum values (Fig. $4 \mathrm{~A}$ and 5A). Changes in the trajectories of the metabolic profiles at different time points may indicate a disordered process in the model, showing a time-dependent change in the urinary metabolites. To acquire differences between the control and the model group and to determine the variables, a supervised pattern recognition method, the OPLS-DA analysis method, was further utilized for metabolic data (Fig. 4B and $5 \mathrm{~B})$.

\section{Metabolites identification}

Chromatographic peaks were abstracted for detection to identify low molecular weight metabolites associated with the disease model. The precise molecular mass was acquired as well as the potential element composition of the compounds, which were shown to possess a degree of unsaturation and fractional isotope abundance within a reasonable error range. The intensity of ions with higher VIP-values (as potential markers) were selected from S-plots and the VIP-plot in positive (Fig. 4C and D) and negative mode (Fig. 5C and D), and a significant difference was observed between the control group and the model group. Finally, a VIP value was selected from the OPLS-DA with a threshold of 1.0, and $P$ values less 
Table 1 Urine potential biomarkers related with ECB rats model identified in positive and negative mode ${ }^{a}$

\begin{tabular}{|c|c|c|c|c|c|c|c|c|c|c|c|}
\hline No. & Rt min & $\begin{array}{l}\text { Mass } \\
\text { determined }\end{array}$ & $\begin{array}{l}\text { Calc } \\
\text { mass }\end{array}$ & $\begin{array}{l}\text { Actual } \\
\text { mass }\end{array}$ & $\begin{array}{l}{[\mathrm{M}-\mathrm{H}]^{-} /} \\
{[\mathrm{M}+\mathrm{H}]^{+}}\end{array}$ & $\mathrm{mDa}$ & ppm & $\begin{array}{l}\text { Proposed } \\
\text { composition }\end{array}$ & Postulated identity & HMDB & Trend \\
\hline 1 & 0.47 & 169.05 & 169.0501 & 168.0422 & {$[\mathrm{M}+\mathrm{H}]^{+}$} & -0.1 & -0.6 & $\mathrm{C}_{8} \mathrm{H}_{8} \mathrm{O}_{4}$ & 3,4-Dihydroxymandelaldehyde & HMDB06242 & $\uparrow \Delta$ \\
\hline 3 & 0.76 & 191.0189 & 191.0192 & 192.027 & {$[\mathrm{M}-\mathrm{H}]^{-}$} & -0.3 & -1.7 & $\mathrm{C}_{6} \mathrm{H}_{8} \mathrm{O}_{7}$ & Citric acid & HMDB00094 & $\uparrow \Delta$ \\
\hline 4 & 0.93 & 182.0822 & 182.0817 & 181.0738 & {$[\mathrm{M}+\mathrm{H}]^{+}$} & 0.5 & 2.7 & $\mathrm{C}_{9} \mathrm{H}_{11} \mathrm{NO}_{3}$ & L-Tyrosine & HMDB00158 & $\downarrow \Delta$ \\
\hline 5 & 1.01 & 132.1025 & 132.1025 & 131.0946 & {$[\mathrm{M}+\mathrm{H}]^{+}$} & 0 & 0 & $\mathrm{C}_{6} \mathrm{H}_{13} \mathrm{NO}_{2}$ & L-Leucine & HMDB00687 & $\downarrow \Delta$ \\
\hline 8 & 2.40 & 206.0459 & 206.0453 & 205.0375 & {$[\mathrm{M}+\mathrm{H}]^{+}$} & 0.6 & 2.9 & $\mathrm{C}_{10} \mathrm{H}_{7} \mathrm{NO}_{4}$ & Xanthurenic acid & HMDB00881 & $\uparrow \Delta \Delta$ \\
\hline 9 & 2.72 & 162.0557 & 162.0555 & 161.0476 & {$[\mathrm{M}+\mathrm{H}]^{+}$} & 0.2 & 1.2 & $\mathrm{C}_{9} \mathrm{H}_{7} \mathrm{NO}_{2}$ & 4,6-Dihydroxyquinoline & HMDB04077 & $\uparrow \Delta \Delta$ \\
\hline 10 & 2.82 & 180.0662 & 180.0661 & 179.0582 & {$[\mathrm{M}+\mathrm{H}]^{+}$} & 0.1 & 0.6 & $\mathrm{C}_{9} \mathrm{H}_{9} \mathrm{NO}_{3}$ & Hippuric acid & HMDB00714 & $\uparrow \Delta \Delta$ \\
\hline 11 & 2.82 & 221.093 & 221.0926 & 220.0847 & {$[\mathrm{M}+\mathrm{H}]^{+}$} & 0.4 & 1.3 & $\mathrm{C}_{11} \mathrm{H}_{12} \mathrm{~N}_{2} \mathrm{O}_{3}$ & 5-Hydroxy-L-tryptophan & HMDB00472 & $\downarrow \Delta \Delta$ \\
\hline 12 & 3.26 & 194.082 & 194.0817 & 193.07389 & {$[\mathrm{M}+\mathrm{H}]^{+}$} & 0.3 & 1.5 & $\mathrm{C}_{10} \mathrm{H}_{11} \mathrm{NO}_{3}$ & Phenylacetylglycine & HMDB00821 & $\uparrow \Delta \Delta$ \\
\hline 17 & 6.68 & 345.2067 & 345.2066 & 344.1987 & {$[\mathrm{M}+\mathrm{H}]^{+}$} & 0.1 & 0.3 & $\mathrm{C}_{21} \mathrm{H}_{28} \mathrm{O}_{4}$ & 11-Dehydrocorticosterone & HMDB04029 & $\uparrow \Delta$ \\
\hline 18 & 6.8 & 317.2111 & 317.2117 & 316.2038 & {$[\mathrm{M}+\mathrm{H}]^{+}$} & -0.6 & -1.9 & $\mathrm{C}_{20} \mathrm{H}_{28} \mathrm{O}_{3}$ & 15-Deoxy- $d-12,14-P G J 2$ & HMDB05079 & $\uparrow \Delta$ \\
\hline 19 & 8.03 & 466.3176 & 466.3169 & 465.309 & {$[\mathrm{M}+\mathrm{H}]^{+}$} & 0.7 & 1.5 & $\mathrm{C}_{26} \mathrm{H}_{43} \mathrm{NO}_{6}$ & Glycocholic acid & HMDB00138 & $\downarrow \Delta$ \\
\hline 20 & 8.14 & 331.1919 & 331.1909 & 332.1987 & {$[\mathrm{M}-\mathrm{H}]^{-}$} & 1 & 3 & $\mathrm{C}_{20} \mathrm{H}_{28} \mathrm{O}_{4}$ & 11b-Hydroxyprogesterone & HMDB04031 & $\downarrow \Delta$ \\
\hline
\end{tabular}

${ }^{a} \uparrow \downarrow$ the relative content of ions which is increased or decreased in the urine on model. Compared control group, $\Delta P<0.05, \Delta \Delta P<0.01$.

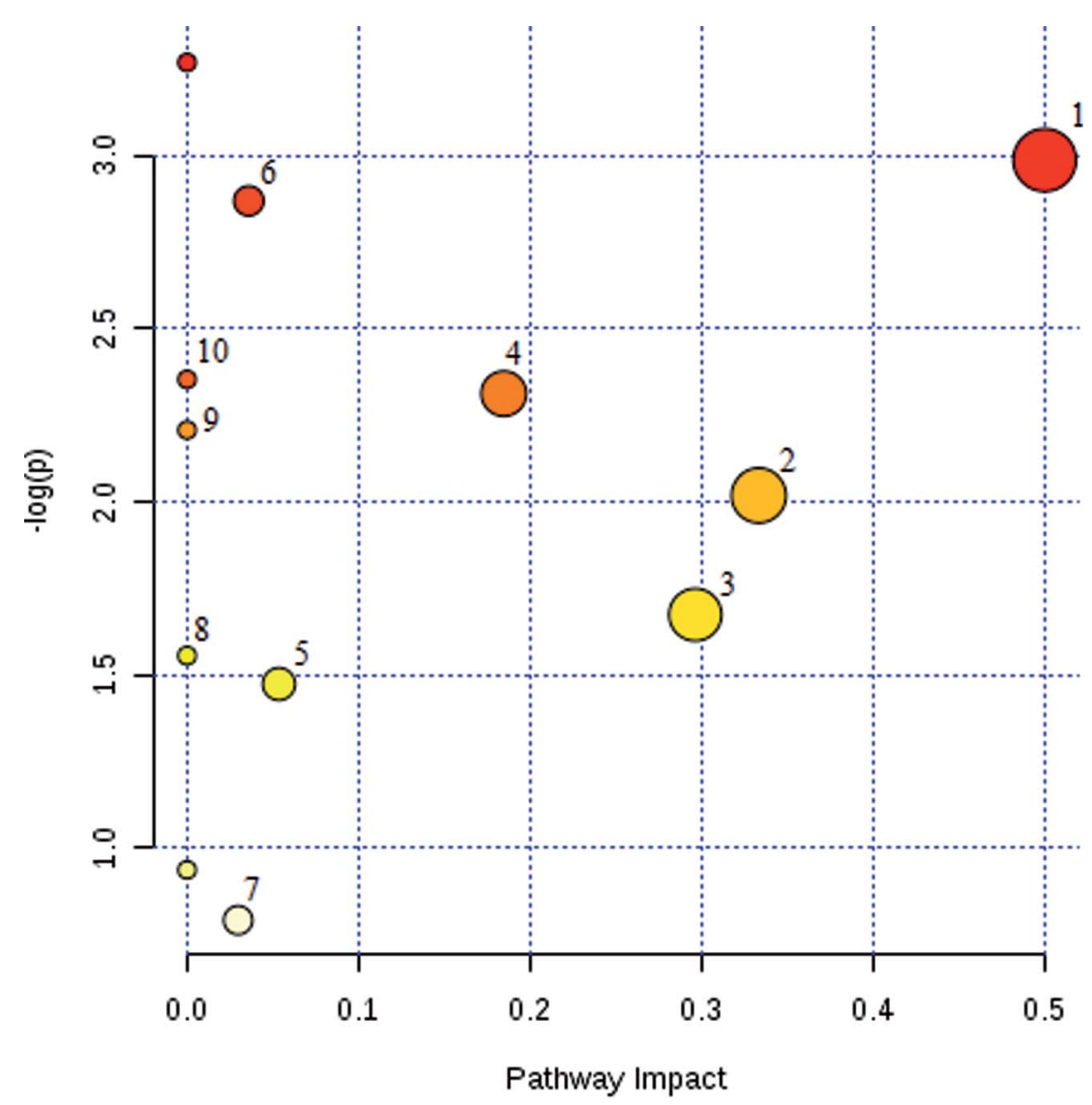

Fig. 6 Summary of pathway analysis with MetaboAnalyst. The ordinate is the original $P$ values obtained from the pathway analysis, and the abscissa is the influence value of pathways obtained from the topological analysis. It is represented by the depth and size of the color of the circle. (1): Phenylalanine, tyrosine and tryptophan biosynthesis; (2): valine, leucine and isoleucine biosynthesis; (3): glyoxylate and dicarboxylate metabolism; (4): tyrosine metabolism; (5): citrate cycle (TCA cycle); (6): steroid hormone biosynthesis; (7): primary bile acid biosynthesis; (8): aminoacyl-tRNA biosynthesis; (9): phenylalanine metabolism; (10): tryptophan metabolism. 

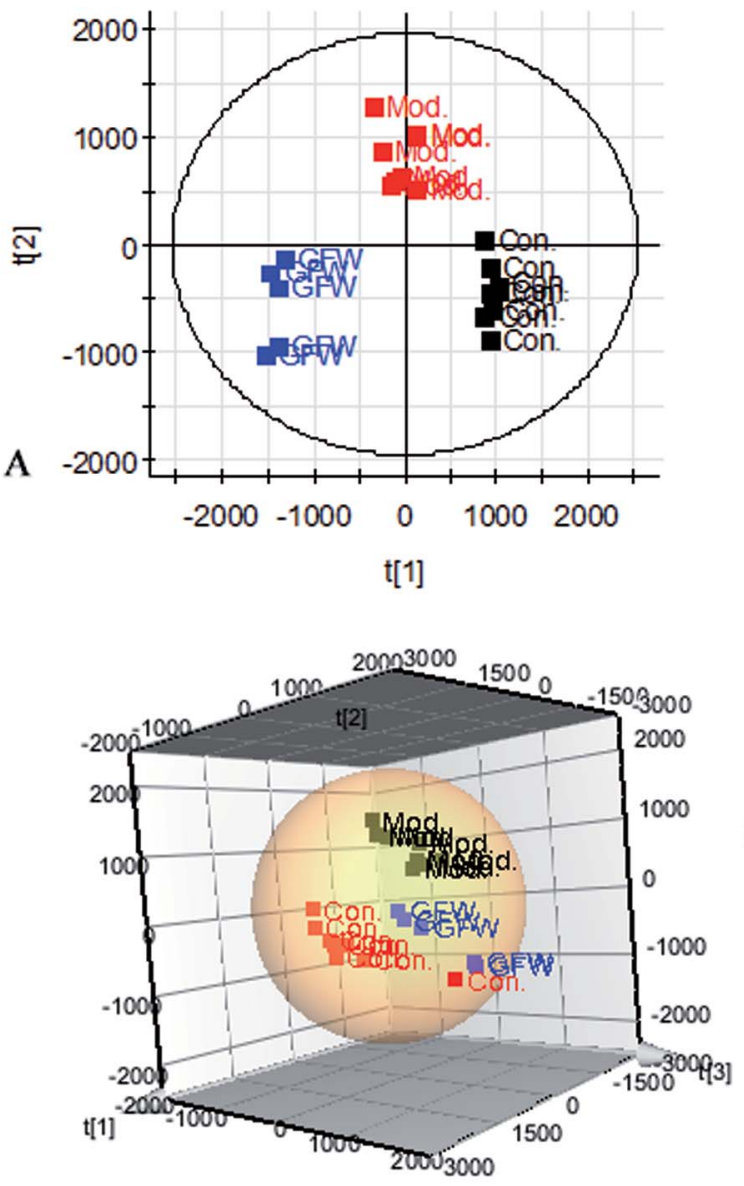

C
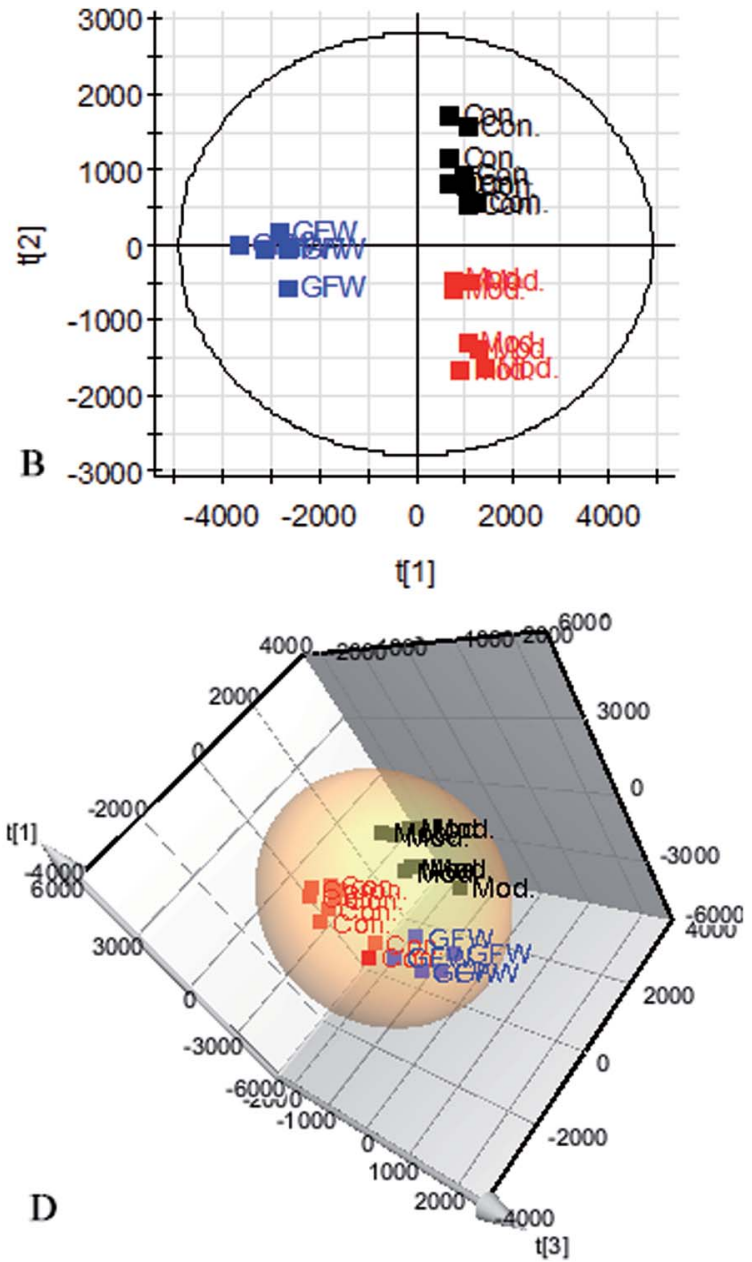

Fig. 7 PLS-DA-score plots of urine profile after oral administration of GFW at 28 days in positive mode (A) and negative mode (B). 3D-PLS-DA score plots of urine profile after oral administration of GFW at 28 days in positive mode (C) and negative mode (D).

than 0.05 were used to identify the candidate biomarker contributing to the clustering between groups. Metabolites with high resolution MS and MS/MS fragments were analyzed further, by searching in databases, HMDB, Chemspider and KEGG. Ultimately, all potential biomarkers associated with each model were identified. In this study, a total of 20 different small molecule metabolites were used to discriminate between the control and model group (4 ions in the negative mode and 16 ions in the positive mode). 7 of the 20 biomarkers were down-regulated with 13 of these being observed to increase (Table 1).

\section{Biological pathway and functional analysis}

The potential pathways and networks for the model were investigated utilizing MetaboAnalyst. MetPA is a free web-based tool with pathway topology analysis that confirms the relevant pathways related to the conditions being studied. Based on the MetPA analysis, it was shown (Fig. 6) that the related metabolite biomarkers primarily participated in the following pathways: phenylalanine, tyrosine and tryptophan biosynthesis, valine, leucine and isoleucine biosynthesis, glyoxylate and dicarboxylate metabolism, tyrosine metabolism, citrate cycle, steroid hormone biosynthesis, tryptophan metabolism, phenylalanine metabolism, primary bile acid biosynthesis, aminoacyl-tRNA biosynthesis, valine, leucine and isoleucine degradation, ubiquinone and other terpenoid-quinone biosynthesis. IPA generated 12 networks. The results imply over the time-course of ECB that these target pathways showed marked perturbations, which could contribute to development of the disease model.

\section{Therapeutic effects analysis based on metabolite profile}

The aim of the PLS-DA analysis was to study the time trajectory of the metabolic profiles in rat urine. The PLS-DA score plots showed clustering of samples from all three groups after administration of different agents after 28 days, with the GFW group being distinctly separate from the other two groups (Fig. 7A and B). This suggests that GFW may have a significant effect on endometriosis rats by changing the anomalous metabolic status, which demonstrated that GFW may be able to 


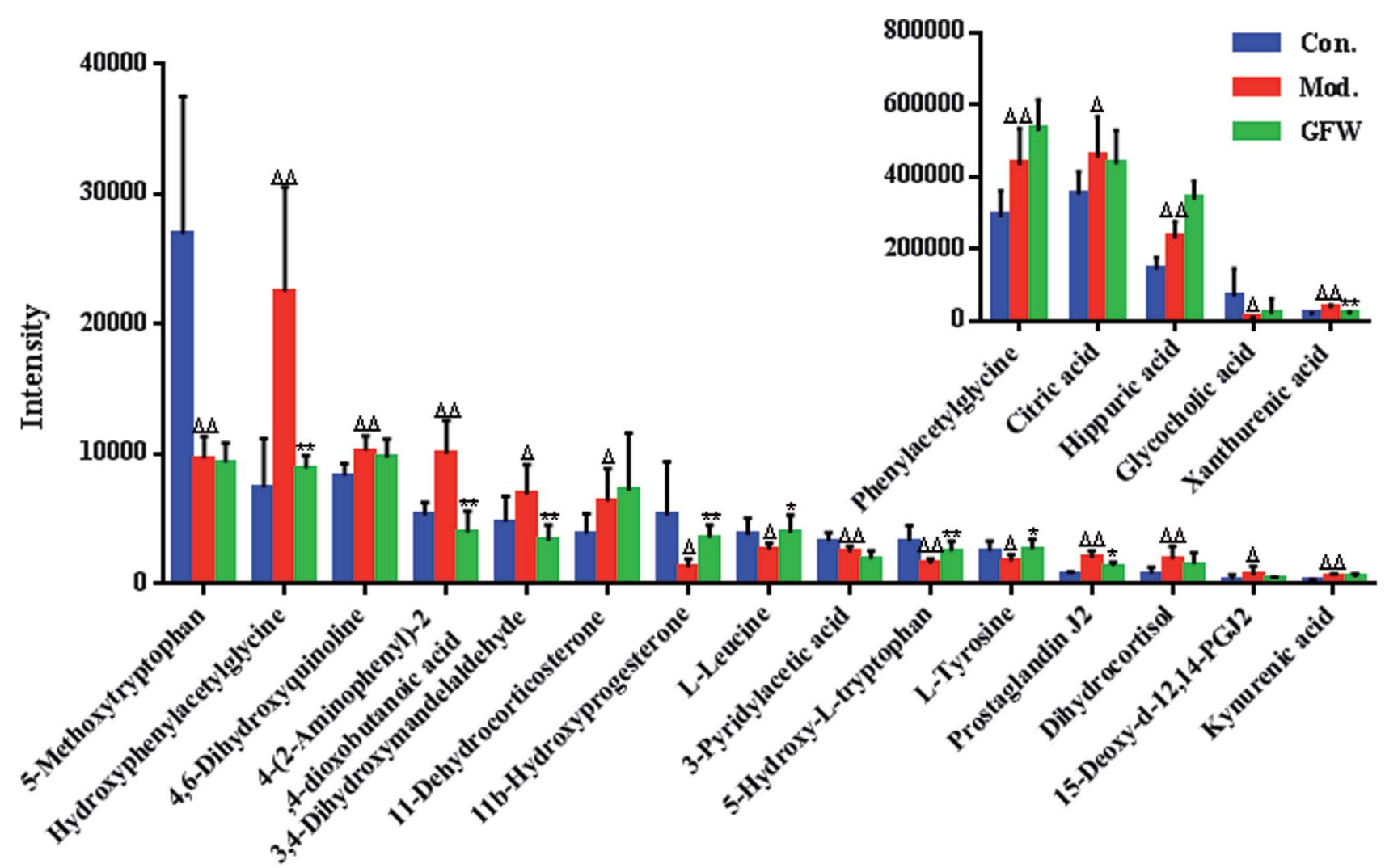

Fig. 8 Relative signal intensities for metabolic biomarkers in the urine, identified in different groups. Model group compared with control group: $\Delta P<0.05, \Delta \Delta P<0.01$; and GFW group compared with model group: $* P<0.05, * * P<0.01$.

improve the pathological process of ECB. The PLS-DA 3D scores in positive and negative mode (Fig. 7C and D), the model and the GFW groups were also clearly separated. By comparing the therapeutic effect of GFW, 15 of biomarkers are obviously reversed, as can be seen in Fig. 8 which shows the average relative content of all biomarkers in the control, model and GFW groups. The intensity of most model-related biomarkers was similar to the normal group following GFW treatment. It was evident that GFW had therapeutic effects on endometriosis through regulating the following main metabolic pathways such as phenylalanine, tyrosine and tryptophan biosynthesis, valine, leucine and isoleucine biosynthesis, glyoxylate and dicarboxylate metabolism, tyrosine metabolism, citrate cycle, steroid hormone biosynthesis, tryptophan metabolism, phenylalanine metabolism, primary bile acid biosynthesis, aminoacyl-tRNA biosynthesis, etc. GFW was shown to have a widespread therapeutic effect on endometriosis by partially regulating the metabolism pathways involve in EBC model (Fig. 9).

\section{Discussion}

Metabolomics is a powerful method for the comprehensive assessments of small molecular weight metabolites of a biological system under transient physiological conditions, and is consistent with the holistic view of traditional Chinese medicine. ${ }^{36}$ Metabolomics uses metabolic profiles to clarify the dynamics of disorders, to interpret the efficacy and toxicity of TCM systematically. GFW was initially recorded in "Correction of Errors in Medical Classics", and its efficacy was demonstrated in a variety of experimental conditions including the decrease of volume of endometrial explants volume. The therapeutic mechanism of GFW is unclear, although its clinical efficacy in the treatment of female endometriosis has been confirmed. In this study, metabolomics was used to explore the pathological metabolic processes and mechanisms of the ECB and to elucidate the therapeutic mechanism of GFW.

The model of endometriosis induced by surgical operation is the classic symptom model. This provides a useful tool to verify the effect of potential therapeutic agents on the proliferation of uterine endometrial tissue. ${ }^{37}$ These autologous endometrial explants resemble human lesions in vivo and function in a similar manner as cells in human endometriosis and in isolated cell cultures. ${ }^{38,39}$ Cold coagulation and blood stasis, as a subtype of blood stasis syndrome, is a major cause of many diseases, including endometriosis and dysmenorrheal in female. In this study, the rat model was established by autologous endometrial transplants and ice-water immersion based on the theory of traditional Chinese medicine. By the $28^{\text {th }}$ day of the study, endometriosis implants were formed entirely. During the process of administration, the ectopic tissue size in model rats did not shrink or even partially increase. Following treatment, the mean volume of implants in GFW group was lower than those in the model group. A histopathology examination 


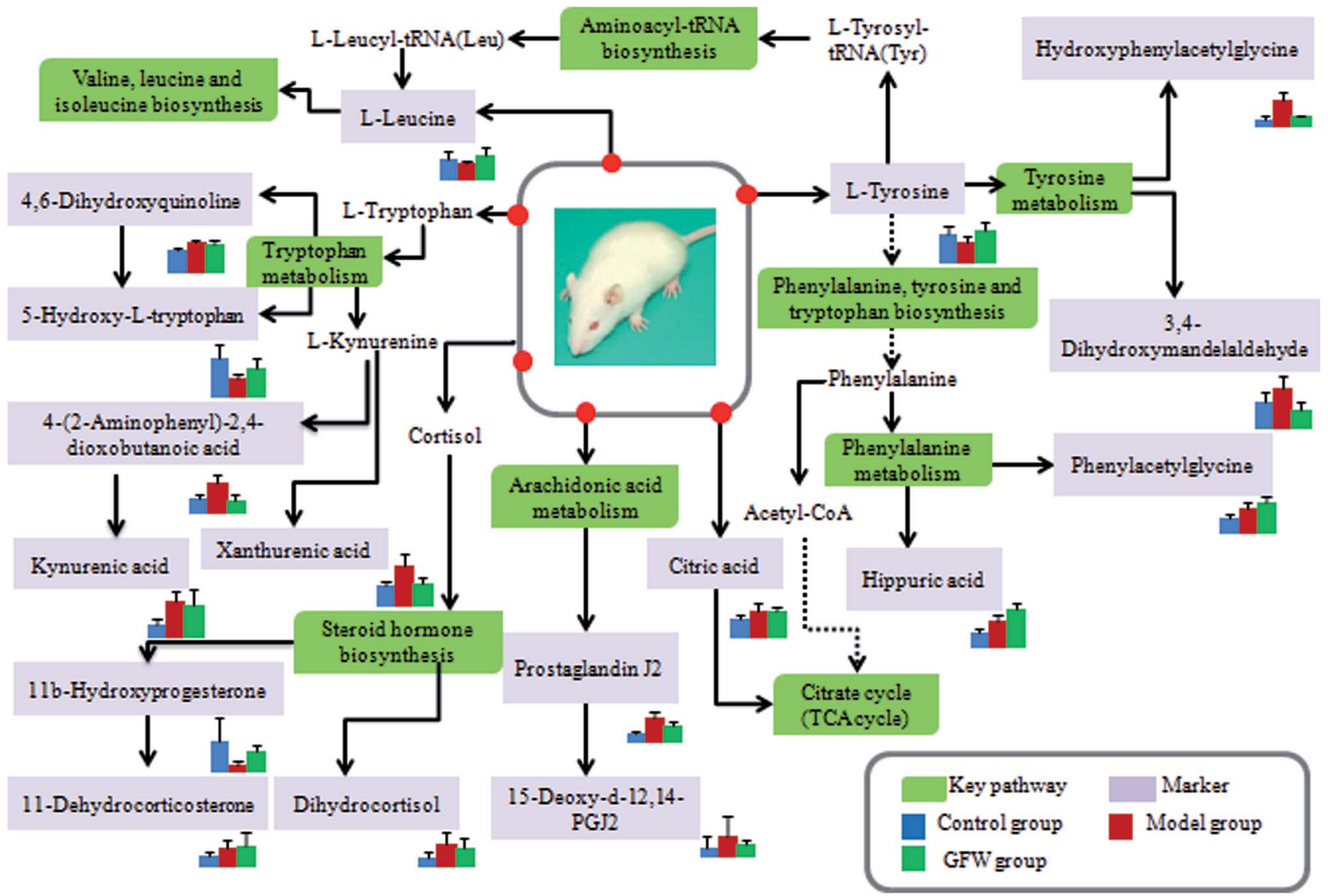

Fig. 9 An overview of the potential disturbed metabolic pathways of endometriosis according to the KEGG pathway metabolic pathway names; light purple boxes mean related metabolites.

showed pathological changes in the ectopic endometrial tissue. Related studies have shown that pathological changes occurring with varying degree of ectopic endometrial tissue provide the mechanism for endometriosis. After the rats with endometriosis were administrated with GFW, the relevant indicators go back to the normal level, illustrating the mechanism of the GFW.

The purpose of this research was to elaborate the therapeutic effects of GFW acting on ECB based on metabolomics and to elucidate the mechanism of endometriosis. Our results demonstrate the therapeutic effect of GFW on the metabolic characteristics of ECB. The rat model was created by autologous endometrial transplants and ice-water immersion to reproduce endometriosis of blood stasis due to the cold accumulation model. Rats were intra-gastrically administered with GFW for 28 consecutive days. By using the supervised pattern recognition method (PLS-DA) based on metabolic patterns, the therapeutic effect of GFW on endometriosis rats could be explained. Compared with the control, the level of 20 metabolites showed significant changes. 7 of 20 biomarkers were down-regulated and 13 of these increased. However, the intensity of the abnormal metabolites of the above-mentioned returned to levels similar to control groups after treatment with GFW (Fig. 8).
Phenylalanine is an aromatic amino acid which is essential for the human body. It is incorporated within various proteins in the body tissue cells, but its oxidation to tyrosine is catalyzed by phenylalanine hydroxylase in the body ${ }^{40}$ Phenylalanine and tyrosine are both used in the synthesis of important neurotransmitters and hormones such as dopamine, epinephrine and norepinephrine. Therefore, a stable phenylalanine metabolic state is critical to maintaining the normal growth and development and normal physiological functions of the body. The metabolism of phenylalanine as well as factors such as liver and kidney disease, affecting the genetic, immune, nervous and endocrine systems will lead to its conversion to tyrosine being blocked, triggering physiological dysfunction.

In this study, the ECB rat model was induced by surgery combined with the ice-water immersion method. Under the condition of blood stasis, due to the large quantity expression of tyrosine hydroxylase and dopamine- $\beta$-hydroxylase, 2 kinds of synthetic rate-limiting enzymes increased the production of catecholamines. The synthesis increased the amounts of epinephrine and norepinephrine, leading to vasoconstriction, thereby increasing blood viscosity and causing blood stasis. The phenylalanine and tyrosine metabolic pathways were disrupted in the endometriosis rats model, in which the $\mathrm{L}$-tyrosine decreased and the hydroxyphenylacetylglycine and 3,4-dihydroxymandelaldehyde abnormally increased. Potential 
biomarkers of hippuric acid and phenylacetylglycine are associated with the phenylalanine metabolic pathway. Up to now, the relationship between the phenylalanine metabolic pathway and inflammation has been reported in the literature, and it has been found that the level of phenylalanine expression increases during body inflammations. The results show that increases in hippuric acid and phenylacetylglycine in the model group was caused by the abnormal metabolism of phenylalanine, which was also one of the reasons leading to aberrations in the tyrosine metabolic pathway.

Tryptophan is an essential amino acid and is the basis of protein biosynthesis. Tryptophan is involved in two main metabolic pathways: the kynurenine pathways and serotonin (5HT) formation. ${ }^{41}$ The kynurenine metabolic pathway (KP) is a significant pathway of tryptophan metabolism, involving the catalysis of the formation of some cascade by indoleamine 2,3two oxidase (IDO), based on the inflammatory factor, which affects the function of neurotransmitters and neural circuits. The principal function of KP is to promote glycolysis, inhibit gluconeogenesis, provide necessary material and energy support for brain tissue, and is especially important for the protection of neurons during periods of inflammation and cytotoxicity. In the occurrence of inflammation, the kynurenine metabolic pathway invokes metabolic disorder, causing significant changes to occur to the kynurenic acid, xanthurenic acid, and 4-(2-aminophenyl)-2,4-dioxobutanoic acid levels. 5Hydroxy-L-tryptophan is a chemical intermediate in the metabolic process of 5-HT. 5-HT is an important messenger and neuromodulator in the body, but also forms platelet factor and neurohormones, and causing a strong contraction of blood vessels. Binding of $5-\mathrm{HT}_{1}$ receptors present in the central nervous system reduces vascular resistance and decreases blood pressure. When 5-HT binds to the $5-\mathrm{HT}_{2}$ receptors distributed on the peripheral vascular smooth muscle, the vasoconstriction and the blood pressure increase. Tryptophan's metabolites, 4,6dihydroxyquinoline and 5-hydroxy--t-tryptophan, were markedly changed in the urine of endometriosis model rats. Administration of GFW significantly alleviated the abnormal function of tryptophan metabolism, tyrosine metabolism and phenylalanine metabolism.

Lipids are the general term for oils, fats and lipids. They are essential nutrients in the human body and one of the core components of the biofilm. Lipids play a significant role in providing energy for the body. Lipid metabolism includes primary bile acid biosynthesis, steroid hormone biosynthesis and arachidonic acid metabolism. Inflammation is a defensive response of living tissue when tissue damage occurs in the vascular system or when pathogenic factors invade the body. It can cause localised swelling, heat, pain and dysfunction. The inflammatory local tissue can be metamorphosed, exuded and proliferated. The arachidonic acid metabolic pathway is a significant part of the inflammatory metabolism network, and the acid can be metabolized to prostaglandins (PGs), thromboxane (TXs), leukotrienes (LTs) and other metabolites, which play an major role in inflammation and are closely related to oxidative stress. When inflammation occurs, PGEs can increase the permeability of venules and capillaries, indirectly stimulating the release of histamine and bradykinin. This enhances the permeability of blood vessels to PGEs. However, the development of inflammation is not related to the total amount of PG, but rather to an imbalance between different types of PG. Prostaglandin J2 (PGJ2) is an endogenous product of mortal inflammation. It causes neuronal death and the accumulation of ubiquitously proteins into distinct aggregates. In a series of events that cause neuronal cell death, PGJ2 may play a role in neurodegenerative diseases. Peroxisome proliferation-activated receptor $\gamma$ (PPAR $\gamma$ ) plays a significant role in glucose homeostasis and adipocyte differentiation. Apart from linoleate and arachidonate, prostaglandin D2 and 15deoxy- $d$-12-14-prostaglandin J2 of PGJ2 natural derivative are the most important PPAR ligands. 15-Deoxy- $d$-12,14-PGJ2 is a metabolite of the PGJ2 prostaglandin family that affects a variety of signaling pathways through the covalent attachment of key signaling molecules. Accumulations of these are largely accompanied by free radicals and the accumulation of reactive oxygen species (including hypertension, asthma and ischemiareperfusion injury). Hence, PGJ2 prostaglandins are often used as markers of oxidative stress. An increase in the intensity of prostaglandin J2 (PGJ2) and 15-deoxy- $d$-12,14-PGJ2 in the urine of endometriosis models may be closely related to the dysfunction ion the arachidonic acid metabolic pathway.

Metabolomics, an emerging science, contributes to the identification of potential biomarkers by analyzing changes in metabolic profiles. ${ }^{42-47}$ In this study, the metabolic patterns of endometriosis rat model were followed to better understand what occurs during treatment with GFW. 20 differential metabolites were found to be associated with endometriosis, involving 12 metabolic pathways. The results show that the therapeutic efficacy of GFW on endometriosis may including follow metabolism of phenylalanine, tyrosine and tryptophan biosynthesis, valine, leucine and isoleucine biosynthesis, glyoxylate and dicarboxylate metabolism, tyrosine metabolism, citrate cycle, steroid hormone biosynthesis, tryptophan metabolism, phenylalanine metabolism, primary bile acid biosynthesis, aminoacyl-tRNA biosynthesis.

\section{Conclusion}

The anti-inflammatory action and metabolic characteristics of GFW was elucidated using surgery combined with ice-water immersion model and a metabolic approach based on UPLCMS coupled with multivariate statistical analysis. 20 biomarkers were examined and the major metabolite network associated with endometriosis explored, and GFW was shown to be effective as treatment for endometriosis by regulating the perturbed metabolism pathways. The primary pathways affected in ECB included: phenylalanine, tyrosine and tryptophan biosynthesis, valine, leucine and isoleucine biosynthesis, glyoxylate and dicarboxylate metabolism, tyrosine metabolism, citrate cycle, steroid hormone biosynthesis, tryptophan metabolism, phenylalanine metabolism, primary bile acid biosynthesis, and aminoacyl-tRNA biosynthesis etc. The study therefore contributed to elucidation of the mechanism of 
endometriosis and the therapeutic mechanism of GFW by an UPLC-MS based metabolism technology for the first time.

\section{Conflicts of interest}

There are no conflicts to declare.

\section{Acknowledgements}

This study was financially supported by National Natural Science Foundation of China (Grant No. 81373931, 81573877, 81430093) and by Innovative Talent Supporting Plan of Heilongjiang University of Chinese Medicine (Grant No. 2012RCD07 and 2012jc01).

\section{References}

$1 \mathrm{X}$. Wang, H. Sun, A. Zhang, et al. Potential role of metabolomics apporoaches in the area of traditional Chinese medicine: as pillars of the bridge between Chinese and Western medicine, J. Pharm. Biomed. Anal., 2011, 55(5), 859-868.

2 W. J. Miao, Q. Wang, T. Bo, et al. Rapid characterization of chemical constituents and rats metabolites of the traditional Chinese patent medicine Gegen-Qinlian-Wan by UHPLC/DAD/qTOF-MS, J. Pharm. Biomed. Anal., 2013, 72(2), 99-108.

3 X. Wang, A. Zhang and H. Sun, Future Perspectives of Chinese Medical Formulae: Chinmedomics as an Effector, OMICS: J. Integr. Biol., 2012, 16(7-8), 414.

4 T. Xue and R. Roy, Studying Traditional Chinese Medicine, Science, 2003, 300(5620), 740.

5 K. S. Lee, Y. T. Lim, S. T. Oh, et al. Modulation of neutrophil apoptosis by plasma and peritoneal fluid from patients with advanced endometriosis, Fertil. Steril., 2002, 77(3), 595-600.

6 M. T. Beste, N. Pfäffledoyle, E. A. Prentice, et al. Molecular Network Analysis of Endometriosis Reveals a Novel Role for c-Jun Regulated Macrophage Activation, Sci. Transl. Med., 2014, 6(222), 222 ra16.

7 I. C. Jeung, K. Cheon and M. R. Kim, Decreased Cytotoxicity of Peripheral and Peritoneal Natural Killer Cell in Endometriosis, BioMed Res. Int., 2016, 2016(2), 1-6.

8 L. Schulke and M. F. Berbic, Dendritic cell populations in the eutopic and ectopic endometrium of women with endometriosis, Hum. Reprod., 2009, 24(7), 1695-1703.

9 A. Hever, R. B. Roth, P. Hevezi, et al. Human endometriosis is associated with plasma cells and overexpression of $\mathrm{B}$ lymphocyte stimulator, Proc. Natl. Acad. Sci. U. S. A., 2007, 104(30), 12451-12456.

10 H. N. Ho, M. Y. Wu, K. H. Chao, et al. Peritoneal interleukin10 increases with decrease in activated CD4+ T lymphocytes in women with endometriosis, Hum. Reprod., 1997, 12(11), 2528-2533.

11 R. N. Taylor, D. I. Lebovic and M. D. Mueller, Angiogenic factors in endometriosis, Ann. N. Y. Acad. Sci., 2002, 955(1), 89-100.
12 T. Harada, T. Iwabe and N. Terakawa, Role of cytokines in endometriosis, Fertil. Steril., 2001, 76(1), 1-10.

13 B. D. Mckinnon, D. Bertschi, N. A. Bersinger, et al. Inflammation and nerve fiber interaction in endometriotic pain, Trends Endocrinol. Metab., 2015, 26(1), 1-10.

14 C. Fox, S. Morin, J. W. Jeong, et al. Local and systemic factors and implantation: what is the evidence?, Fertil. Steril., 2016, 105(4), 873.

15 T. E. Sensky and D. T. Liu, Endometriosis: associations with menorrhagia, infertility and oral contraceptives, Int. J. Gynaecol. Obstet., 1980, 17(17), 573-576.

16 F. Liao, Herbs of activating blood circulation to remove blood stasis, Clin. Hemorheol. Microcirc., 2001, 23(suppl. 1), 127.

17 X. J. Ma, H. J. Yin and K. J. Chen, [Investigation of gene expression profiles in patients with blood stasis syndrome], J. Chin. Integr. Med., 2008, 6(4), 355-360.

18 F. Amant, P. Moerman, P. Neven, et al. Endometrial cancer, Lancet, 2005, 366(9484), 491-505.

19 M. W. Laschke and M. D. Menger, Anti-angiogenic treatment strategies for the therapy of endometriosis, Hum. Reprod. Update, 2012, 18(6), 682-702.

20 R. L. Barbieri, Hormone treatment of endometriosis: the estrogen threshold hypothesis, Am. J. Obstet. Gynecol., 1992, 166(2), 740-745.

21 A. H. Zhang, J. B. Yu, H. Sun, L. Kong, X. Q. Wang, Q. Y. Zhang and X. J. Wang, Identifying quality-markers from Shengmai San protects against transgenic mouse model of Alzheimer's disease using chinmedomics approach, Phytomedicine, 2018, S0944-7113(18), 30104.

22 A. Zhang, H. Sun, Y. Han, et al. Exploratory urinary metabolic biomarkers and pathways using UPLC-Q-TOFHDMS coupled with pattern recognition approach, Analyst, 2012, 137(18), 4200-4208.

23 N. Yang, X. Zhou, L. Qi, et al. Serum metabolomics strategy for understanding pharmacological effects of ShenQi pill acting on kidney yang deficiency syndrome, J. Chromatogr. B: Anal. Technol. Biomed. Life Sci., 2015, 1026, 217.

24 A. Zhang, H. Fang, Y. Wang, et al. Discovery and verification of the potential targets from bioactive molecules by network pharmacology-based target prediction combined with highthroughput metabolomics, RSC Adv., 2017, 7, 51069-51078.

25 G. A. Canuto, E. A. Castilhomartins, M. F. Tavares, et al., Multi-analytical platform metabolomic approach to study miltefosine mechanism of action and resistance in Leishmania, Anal. Bioanal. Chem., 2014, 406(14), 3459-3476.

26 H. Chu, A. H. Zhang, Y. Han, et al. Metabolomics and its potential in drug discovery and development from TCM, World Journal of Traditional Chinese Medicine, 2015, 1, 26-32.

27 A. Zhang, H. Sun and X. Wang, Mass spectrometry-driven drug discovery for development of herbal medicine, Mass Spectrom. Rev., 2018, 37(3), 307-320.

28 A. Zhang, X. Zhou, H. Zhao, et al. Metabolomics and proteomics technologies to explore the herbal preparation affecting metabolic disorders using high resolution mass spectrometry, Mol. BioSyst., 2017, 13(2), 320-329. 
29 F. Zhou, T. W. Sikorski, S. B. Ficarro, et al. An Online Nanoflow RP-SAX-RP LC-MS/MS Platform for Efficient and In-depth Proteome Sequence Analysis of Complex Organisms, Anal. Chem., 2011, 83(18), 6996-7005.

30 S. Chen, C. Xiong, H. Liu, et al. Mass spectrometry imaging reveals the sub-organ distribution of carbon nanomaterials, Nat. Nanotechnol., 2015, 10(2), 176-182.

31 H. Sun, L. Yang, M. X. Li, et al., UPLC-G2Si-HDMS untargeted metabolomics for identification of metabolic targets of Yin-Chen-Hao-Tang used as a therapeutic agent of dampness-heat jaundice syndrome, J. Chromatogr. B: Anal. Technol. Biomed. Life Sci., 2018, 1081-1082, 41-50.

32 X. Wang, A. Zhang, L. Kong, et al. Rapid discovery of qualitymarkers from Kaixin San using chinmedomics analysis approach, Phytomedicine, 2017, S0944-7113(17), 30188.

33 W. W. Ma, A. H. Zhang, H. Sun, et al. Metabolomics Deciphering Therapeutic Effects and Mechanism of Traditional Chinese Medicines, World Journal of Traditional Chinese Medicine, 2016, 2(3), 17-24.

$34 \mathrm{H}$. Chu, H. Sun, G. Yan, et al. Metabolomics analysis of health functions of Physalis pubescens L. using by ultraperformance liquid chromatography/electrospray ionization quadruple time-of-flight mass spectrometry, World Journal of Traditional Chinese Medicine, 2015, 1(3), 112.

35 M. W. Vernon and E. A. Wilson, Studies on the surgical induction of endometriosis in the rat, Fertil. Steril., 1985, 44(5), 684-694.

36 S. J. Mihalik, S. F. Michaliszyn, H. J. De, et al. Metabolomic profiling of fatty acid and amino acid metabolism in youth with obesity and type 2 diabetes: evidence for enhanced mitochondrial oxidation, Diabetes Care, 2012, 35(3), 605.

37 M. W. Vernon and E. A. Wilson, Studies on the surgical induction of endometriosis in the rat, Fertil. Steril., 1985, 44(5), 684-694.
38 K. L. Sharpe-Timms, Using rats as a research model for the study of endometriosis, Ann. N. Y. Acad. Sci., 2002, 955(1), 318-327.

39 I. Uchiide, T. Ihara and M. Sugamata, Pathological evaluation of the rat endometriosis model, Fertil. Steril., 2002, 78(4), 782-786.

$40 \mathrm{H}$. Watanabe, Y. Miyamoto, M. Otagiri, et al. Update on the pharmacokinetics and redox properties of protein-bound uremic toxins, J. Pharm. Sci., 2011, 100(9), 3682-3695.

41 X. H. Zhou, A. H. Zhang, L. Wang, et al. Novel chinmedomics strategy for discovering effective constituents from ShenQiWan acting on ShenYangXu syndrome, Chin. J. Nat. Med., 2016, 14(8), 561-581.

42 A. Zhang, Q. Liu, H. Zhao, et al. Phenotypic characterization of nanshi oral liquid alters metabolic signatures during disease prevention, Sci. Rep., 2016, 6, 19333.

43 A. Zhang, H. Sun, G. Yan, et al. Mass spectrometry-based metabolomics: applications to biomarker and metabolic pathway research, Biomed. Chromatogr., 2016, 30(1), 7-12.

44 A. H. Zhang, H. Sun, S. Qiu, et al. NMR-based metabolomics coupled with pattern recognition methods in biomarker discovery and disease diagnosis, Magn. Reson. Chem., 2013, 51(9), 549-556.

45 A. Zhang, H. Sun, P. Wang, et al. Metabonomics for discovering biomarkers of hepatotoxicity and nephrotoxicity, Die Pharmazie-An International Journal of Pharmaceutical Sciences, 2012, 67(2), 99-105.

$46 \mathrm{H}$. Chu, A. Zhang, Y. Han, et al. Metabolomics approach to explore the effects of Kai-Xin-San on Alzheimer's disease using UPLC/ESI-Q-TOF mass spectrometry, J. Chromatogr. B: Anal. Technol. Biomed. Life Sci., 2016, 1015, 50-61.

47 A. Zhang, G. Yan, X. Zhou, et al. High resolution metabolomics technology reveals widespread pathway changes of alcoholic liver disease, Mol. BioSyst., 2016, 12(1), 262-273. 